\title{
An electrostatic storage ring for atomic and molecular physics, at KACST - a status report
}

\author{
Mohamed O.A. El Ghazaly \\ Physics and Storage of Molecular Ions, National Center for Mathematics and Physics (NCMP) King \\ Abdulaziz City for Science and Technology (KACST), PO Box 6086, Riyadh 11442, Saudi Arabia
}

\begin{abstract}
An electrostatic storage ring has been designed following the pioneering storage ring ELISA [1], and it is currently being built as a new core laboratory for atomic and molecular collisions at the King Abdulaziz City for Science and Technology (KACST), in Riyadh, Saudi Arabia. In this paper, the design of the electrostatic storage ring together with an outline on the status of the construction are given.
\end{abstract}

\section{Introduction}

Advance in research has long been associated with the emergence and development of state-of-the-art techniques and innovative apparatus. Indeed, ground-breaking advances in collisions physics have been realized over the last decades, and key to these advances has been the use and ability of the storage of electrons or ions in magnetic rings. In particular, ion storage rings have been used for their ability to produce high-quality beams of internally cold molecular ions [2]. Thus, heavy-ion storage rings have over time proven to be an outstanding tool in atomic and molecular physics. They have been used, in electron-ion collision experiments, in combination with very sophisticated electron coolers that provide high-current electron beams. This state-of-the art combination has been implemented at CRYRING [3], ASTRID [4], TSR [5], and TARN II [6] magnetic storage rings, in a merged electron-ion beam configuration [7]. This is suitable for investigating dissociative recombination (DR) or similar low-energy reactions as well as the process of dissociative excitation (DE). Indeed, the mergedbeams method enables the collision between the electron and molecular ion to occur at low relative energies. That is, in the energy domain where DR and similar low-energy reactions prominently take place. Similar to DR is e.g. the Resonant positive-negative Ion Pair formation (RIP), in which the recombination stabilizes through the formation of a charged ion pair. Higher up in energy, Dissociative Excitation (DE) begins to dominate electron-molecular ion interactions above a few electron-volts, an energy domain where DR decreases in efficiency. DR and similar low-energy reactions together with $\mathrm{DE}$ are fundamental reactions in low temperature plasmas whether industrial or astrophysical. They have been, therefore, attracting extensive interest in terms of both theory and experiment. Further development of associated techniques and devices is still taking place. Nevertheless, magnetic rings

${ }^{a}$ Corresponding author: maelghazaly@kacst.edu.sa

This is an Open Access article distributed under the terms of the Creative Commons Attribution License 4.0, which permits unrestricted use, distribution, and reproduction in any medium, provided the original work is properly cited. 
inherit the intrinsic disadvantages of the magnetic field such as the field rigidity, which restricts the domain of application of such rings to light molecular ions.

Recently, heavy-ion electrostatic storage rings have been introduced as mass-independent storage devices that are thereby suitable for investigating heavy molecular ion reactions. Hence, electrostatic storage rings complement the purpose of their magnetic counterparts by opening up the field to heavier species such as those encountered in the biological sciences. ELISA (ELectrostatic Ion Storage ring, Aarhus) [1] was the first electrostatic storage ring dedicated to the storage of heavy ions. It features a racetrack shaped ring of $\sim 7 \mathrm{~m}$ circumference, with an operating energy in the range of 20-25 q. $\mathrm{keV}$. Since this initial design and implementation, the KEK-ring [8] and the TMU E-ring [9] have given further impetus to the field. They share an identical racetrack layout and ion-optical lattice. Also they have a similar size, and operate at the same range of energy as that of ELISA. One refers to this as the standard or ELISA-like design. Other room-temperature or cryogenically-cooled, single or double electrostatic storage rings are about to be or are being completed [10]. Smaller and miniature electrostatic rings have also been built or are currently under development, see e.g., Ref. [10]. In addition, design studies have been or are being carried out to optimize and further the development of electrostatic storage rings. Indeed, a design for an Ultra-low energy electrostatic Storage Ring (USR) has been carried out for the Facility for low-energy Antiproton and Ion Research (FLAIR), at GSI, Germany [11]. Another optimized design for a specific electrostatic storage ring to be added to the utility of these storage devices is being launched at the Jet Propulsion Laboratory [12].

So far, ELISA, KEK-ring, and TMU E-ring are the only standard-sized electrostatic storage rings in operation. Nevertheless, they have been driving cutting-edge research programs in the interdisciplinary fields of heavy ions of relevance to biophysics, biochemistry, radio-biology and even bio-astrophysics. Certainly, the focus of such experimental research program ranges from lifetime measurements of multiply charged metastable states and dynamics of clusters, to photo-excitation of photoactive protein chromophores [13-17]. Moreover, with an electron target (ETRAP), in a crossed-beam configuration on ELISA and a small electron cooler in a merged-beam combination with KEK ring, these rings have initiated ground-breaking experiments on electron-impact induced reactions in heavy molecular ions. The focus was dominated by the DR and DE of biomolecular ions and clusters, along with Electron Detachment (ED) or Electron Attachment (EA) to multiply-charged ions [18-22].

Recently, a comprehensive design study for a low-deflecting electrostatic storage ring, a $7^{\circ}$ design [23], has been carried out, in the course of the project for building a storage ring facility for atomic and molecular collisions, at KACST in Riyadh, Saudi Arabia [24]. The design of the ring of such facility is rather optimized with regards to the standard design. The present paper provides an overview of the scientific motivations of this project, and reports on the status of this electrostatic storage ring that is being built as the core of such a facility.

\section{Motivations}

The challenging idea to develop and build from scratch an up-to-date complete storage ring facility at KACST, was launched through an ambitious proposal by the author [24] to develop the first atomic and molecular physics laboratory in Saudi Arabia. The ultimate objective through this project is to form a local community for atomic and molecular collisions. Another objective is to introduce and transfer the electrostatic storage know-how to the local scientific community. Indeed, in the course of the project, several young researchers have been introduced to, taught and trained on the development of electrostatics storage rings [25-27]. One recent outcome of this project has been an excellent M.Sc. thesis that includes the $7^{\circ}$ design $[28,29]$.

The purpose of this storage ring facility is to provide the experimental infrastructure needed to continue and further develop those types of experiments performed previously in doctoral [30] and postdoctoral [20] research in the field of electron-ion and photon-ion interactions. Thus, the conceptual 
design and structure of this facility combine an electrostatic storage ring similar to the pioneering storage ring ELISA $[1,20]$ and a flexible ion injector that also features a single-pass set-up similar to the welltested electron-ion single-pass experiment in Louvain, Belgium [30, 31]. Such a combination is expected to enable absolute measurement of cross-sections for various electron-ion collision processes, such as DR, RIP or other similar low-energy reactions.

\section{Concept and design of the electrostatic storage ring}

Above all its advantages, the merit of an electrostatic storage ring goes first to the fact that it has no limitation on the mass of the ions that can be stored. The electrostatic field $(E)$ that is required to bend the trajectory of a particle with charge $q$ is proportional to the quantity $T / q \rho$; where $T$ and $\rho$ are the kinetic energy of the particle and the bending radius of the trajectory, respectively. Since $T$ is initially defined by the acceleration voltage $\left(\Delta U_{A c c}\right)$ of the high voltage (HV)-platform; the electrostatic field rigidity $(\rho E)$ depends only on the kinetic energy of the particle and not its mass:

$$
\rho E=2 \Delta U_{A c c}
$$

In many respects, it is this wonderful property of the electrostatic field that has triggered and driven the transition to the electrostatic rings.

A second merit goes to the concept and the simplicity of an electrostatic storage ring which relies on the exclusive use of electrostatic devices to act on and store the ion beam. Essentially, a distinctive feature of this concept is that it limits itself to linear beam optics. Thus, the lattice of an electrostatic storage ring consists of dipoles to bend the beam, and quadrupoles to focus and shape it, inter-spaced by field-free drift regions. No accelerating or corrective optics (sextupoles or octupoles) have been introduced into the current concept of the ion electrostatic rings. In the standard design, dipole fields are generated by either Cylindrical Deflectors (CD) or Pairs of Deflecting Plates (PDP). In addition, the latter are used for beam injection and extraction. Quadrupoles are used in doublet combinations to ensure net focusing in both transversal planes.

In the standard racetrack layout, the U-turn of the beam is made by a single-bend CD complemented by two identical upstream and downstream PDPs. Hence, the deflecting angle $(\theta)$ in the PDP characterizes the design and singles out this electrostatic storage ring. Indeed, the use of a low deflecting angle, $\theta$, avoids the problems related to the rise time of the field and switching high voltages on the PDP. It also likely to lower the energy-variations and aberration effects, which are inherent in electrostatic deflecting optics. Thus, the $7^{\circ}$ design [23] features an optimization of the electrostatic storage ring design with respect to such effects. On the other hand, a large deflection in a PDP leads to an increase of the angular acceptance of the detection in electrostatic rings, and accordingly the collection of light produced fragments in dissociative processes. The standard design illustrated in ELISA features an optimal deflection in the PDP with a deflecting angle of $\theta=10^{\circ}$, and accordingly a bending angle in the $\mathrm{CD}$ of $\beta=160^{\circ}$. The KACST storage ring has been optimized to dissociative processes, and so it closely follows the standard or ELISA-like design. A 3D illustration of the KACST storage ring as designed by the SolidWork program, is shown in Fig. 1.

\section{Status of the storage ring}

Currently, the whole ring is close to be completed. Indeed, all vacuum chambers have been manufactured together with optical components such as quadrupole doublets, cylindrical deflector set-ups, pairs of deflecting plates. All have been assembled and mounted inside their corresponding chambers. The vacuum pumping system which mainly consists of four ion-sublimation combination pumps is ready. The ring is to be mounted at KACST in the spring of year 2014. Vacuum testing, beam injection and characterization will then be performed, and the first storage of the beam can therefore be expected in 


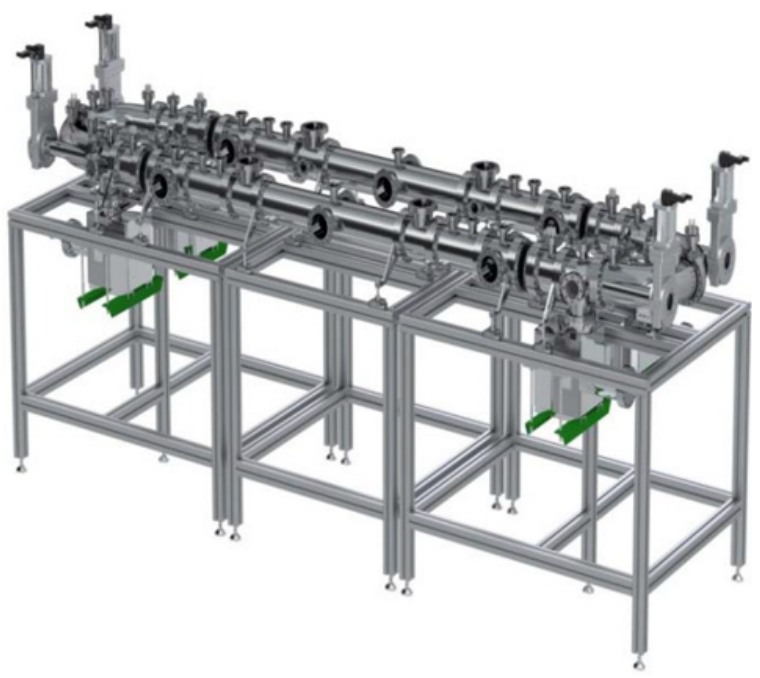

Figure 1. A 3D representation of the KACST storage ring, as designed by SolidWork.

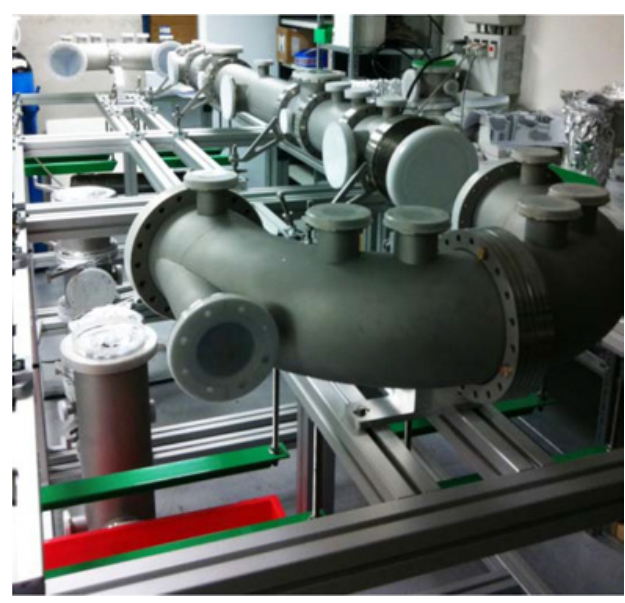

Figure 2. A photograph of the KACST storage ring under construction.

about a semester later. Meanwhile, the beam diagnostic devices to be used to profile and view the ion beam in the ring, is being investigated. The detection and imaging system with an MCP and a phosphor screen are to be ordered.

Furthermore, one can see that there has been always a need for new ideas in a storage ring facility. To this end, therefore, a new conceptual design for a compact type quadrilateral ring has recently been worked out in the frame of a proposal for a new small, transportable electrostatic storage ring [32]. This will be the subject of a later paper.

This project is funded by the King Abdulaziz City for Science and Technology (KACST) under the grant no. 162-28/PI: MOA_El Ghazaly. The author is grateful to KACST for the fund and support to this project. The author gratefully acknowledges the crucial support and welcome offered by $\mathrm{H}$. $\mathrm{H}$. Alharbi, for the project at the 
National Center for Mathematics and Physics, KACST. Thanks to all colleagues who have contributed to this project. P. Defrance and J. B. Mitchell are warmly thanked for valuable discussions and efforts related to this work. Thanks also go to colleagues from Meca2000 of Vinci technologies.

\section{References}

[1] Søren Pape Møller, Nucl. Instr. and Meth. A 394 (1997) 281-286

[2] Mats Larsson, Rep. Prog. Phys. 58 (1995) 1267-1319

[3] K. Abrahamsson, G. Andler, L. Bagge, E. Beebe, P. Carlé, H. Danared, S. Egnell, K. Ehrnstén, M. Engström, C. Herrlander, J. Hilke, J. Jeansson, A. Källberg, S. Leontein, L. Liljeby, A. Nilsson, A. Paal, K.-G. Rensfelt, U. R. rd, A. Simonsson, A. Soltan, J. Starker, M. af Ugglas, and A. Filevich, Nucl. Instrum. Methods Phys. Res. B 79, 269 (1993)

[4] S. P. Møller, Proceedings of the 1991 IEEE Particle Accelerator Conference, edited by L. Lizama and J. Chew (IEEE, New York, 1991), p. 2811

[5] D. Krämer, G. Bisoffi, M. Blum, A. Friedrich, C. Geyer, B. Holzer, H. W. Heyng, D. Habs, E. Jaeschke, M. Jung, W. Ott, R. E. Pollock, R. Repnow, F. Schmitt, and M. Steck, Nucl. Instrum. Methods Phys. Res. A 287, 268 (1990)

[6] T. Tanabe, K. Noda, T. Honma, M. Kodaira, K. Chida, T. Watanabe, A. Noda, S. Watanabe, A. Mizobuchi, M. Yoshizawa, T. Katayama, H. Muto, and A. Ando, Nucl. Instrum. Methods Phys. Res. A 307, 7 (1991)

[7] R. D. Thomas, H. T. Schmidt, G. Andler, M. Björkhage, M. Blom, L. Brännholm, E. Bäckström, H. Danared, S. Das, N. Haag, P. Halldén, F. Hellberg, A. I. S. Holm, H. A. B. Johansson, A. Källberg, G. Källersjö, M. Larsson, S. Leontein, L. Liljeby, P. Löfgren, B. Malm, S. Mannervik, M. Masuda, D. Misra, A. Orbán, A. Paál, P. Reinhed, K.-G. Rensfelt, S. Rosén, K. Schmidt, F. Seitz, A. Simonsson, J. Weimer, H. Zettergren, and H. Cederquist, Rev. Sci. Instrum. 82, 065112 (2011)

[8] T. Tanabe, K. Chiba, K. Noda, I. Watanabe, Nucl. Instr. Meth. A 482 (2002) 595c

[9] S. Jinno, T. Takao, Y. Omata, A. Satou, H. Tanuma, T. Azuma, H. Shiromaru, K. Okuno, N. Kobayashi, I. Watanabe, Nucl. Instr. Meth. A 532 (2004) 477c

[10] H. T. Schmidt, et al. Rev. Sci. Instrum. 84, 055115 (2013)

[11] C.P. Welsch, M. Grieser, J. Ullrich, A. Wolf, Nucl. Instr. and Meth. A 546 (2005) 405c

[12] Chutjian et al.: "Proposal for a New Electrostatic Storage Ring at Jet Propulsion Laboratory" http://www.tapir.caltech.edu/ kamion/CFP/Chutjian_Storage_Ring_and_EBIT_ for_Lab_Astro_SSE_GAN_CFP.pdf

[13] U.V. Pedersen, M. Hyde, S.P. Møller, T. Andersen, Phys. Rev. A 64 (2001) 012503

[14] K. Hansen, J.U. Andersen, P. Hvelplund, S.P. Møller, U.V. Pedersen, V.V. Petrunin, Phys. Rev. Lett. 87 (2001) 123401

[15] S.B. Nielsen, A. Lapierre, J.U. Andersen, U.V. Pedersen, S. Tomita, L.H. Andersen, Phys. Rev. Lett. 87 (2001) 2281021

[16] L.H. Andersen, I.B. Nielsen, M.B. Kristensen, M.O.A. El Ghazaly, S. Haacke, M. Brønsted Nielsen, M. ${ }^{\circ}$ Axman Petersen, Journal of the American Chemical Society 127 (35) (2005) 12347

[17] I.B. Nielsen, S. Boyeí-Peronne, M.O.A. El Ghazaly, M.B. Kristensen, S. Brøndsted Nielsen, L.H. Andersen, Biophysical Journal 89 (2005) 2597

[18] T. Tanabe, J. Phys.: Conf. Ser. 58 (2007) 17

[19] Tetsumi Tanabe, Koji Noda, Evgeny Syresin, Nucl. Instr. Meth. A 532 (2004) 105-110

[20] M.O.A. El Ghazaly, A. Svendsen, H. Bluhme, A.B. Nielsen, S. Brøndsted Nielsen, L.H. Andersen, Phys. Rev. Lett. 93 (2004) 203201

[21] M.O.A. El Ghazaly, A. Svendsen, S.B. Nielsen, and L.H. Andersen Chem. Phys. Lett. 405, 278$281(2005)$ 
[22] A. Svendsen, H. Bluhme, M.O.A. El Ghazaly, K. Seiersen, S.B. Nielsen, L.H. Andersen, Phys. Rev. Lett. 94 (2005) 223401

[23] M.O.A. El Ghazaly, S.M. Alshammari, C.P. Welsch, H.H. Alharbi, Nucl. Instr. Meth. A 709 (2013) 76-84

[24] Mohamed O A El Ghazaly, "KACST ESRing - Status and outlook", the 4th International Workshop on Electrostatic Storage Devices-ESD11, Oak Ridge National Laboratory (ORNL), Tennessee, USA, June 8-11, 2011 http://www-cfadc.phy.ornl.gov/ESD_2011/ abstracts/invited/ElGhazaly.pdf Also: Mohamed O A El Ghazaly: "A Proposal for the Development of a New Electrostatic Storage Ring at KACST", Grant no. 162-28/MOA_El Ghazaly, NCMP, KACST, Riyadh, 2007

[25] M.O.A. El Ghazaly, A.A. Alzeanidi, A. Papash, and C.P. Welsch, J. Applied Mathematics and Information Sciences, 3 (3) 301-307 (2009)

[26] M.O.A. El Ghazaly, M. Al-Malki, A. Papash, C.P. Welsch, Proceeding of the $23^{\text {rd }}$ Particle Accelerator Conference (PAC09), Vancouver, Canada (2009)

[27] M.O.A. El Ghazaly, S.A. Behery, A.A. Almuqhim, A.I. Papash, and C.P. Welsch, AIP Conf. Proc. 1370, $272(2011)$

[28] A.A. Almuqhim, “The KACST Electrostatic Storage Ring: Design \& Simulations”, Master thesis supervised by Mohamed O A El Ghazaly, King Saud University, Riyadh (2011)

[29] A.A. Almuqhim, S.M. Alshammari, M.O.A. El Ghazaly, A.I. Papash, and C.P. Welsch, AIP Conf. Proc. 1370, 278 (2011)

[30] Mohamed O A El Ghazaly, "Dissociation des ions $H_{2}^{+}, D_{2}^{+}$, and $D_{3}^{+}$par impact d'électrons" Ph.D. Dissertation (2003) Université Catholique de Louvain, Belgium

[31] M.O. Abdellahi El Ghazaly, J. Jureta, X. Urbain and P. Defrance, J. Phys. B: At. Mol. Opt. Phys. 37, No. 12 2467-2483 (2004)

[32] Mohamed O.A. El Ghazaly, "Perspective for a compact electrostatic storage ring" XXVIII International Conference on Photonic, Electronic and Atomic Collisions-ICPEAC2013 (2013) http://icpeac2013.impcas.ac.cn/absbook/abstracts/T14\%20Related\%20Topics/ $22691 \cdot p d f$ 